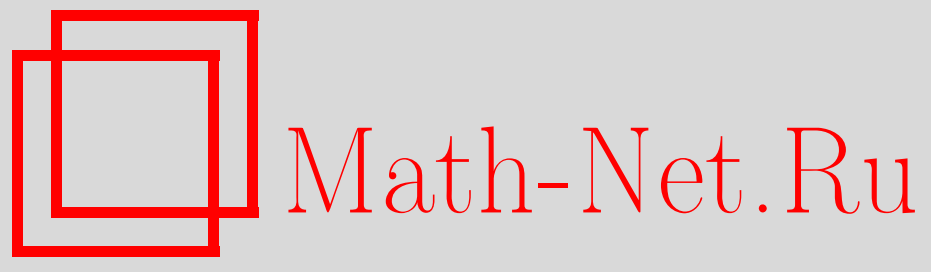

Э. Файрайзл, Динамические системы в моделях механики жидкости, УМН, 2014, том 69, выпуск 2, 149-176

DOI: https://doi.org/10.4213/rm9573

Использование Общероссийского математического портала Math-Net.Ru подразумевает, что вы прочитали и согласны с пользовательским соглашением http: //www . mathnet.ru/rus/agreement

Параметры загрузки:

IP: 54.174 .149 .18

26 апреля 2023 г., 14:50:30

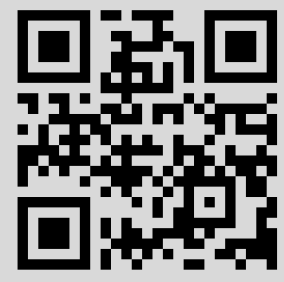




\section{Динамические системы в моделях механики жидкости}

\section{Э. Файрайзл}

В работе дается обзор некоторых современных результатов по асимптотическому поведению решений моделей полных жидких систем при больших временах, при этом в моделях учитываются сжимаемость, вязкость и(или) теплопроводность жидкостей. Мы вводим несколько понятий решения и обсуждаем вопросы существования глобальных по времени траекторий, а также общие вопросы, связанные с корректностью. Рассматриваются свойства диссипативности и, в частности, существование поглощающих множеств и асимптотическая компактность траекторий. Изучаются вопросы существования аттракторов, сходимость к равновесному решению и другие качественные особенности поведения при больших временах.

Библиография: 57 названий.

Ключевые слова: система Навье-Стокса-Фурье, поведение при больших временах, слабое решение.

DOI: $10.4213 / \mathrm{rm} 9573$

\section{СОДЕРЖАНИЕ}

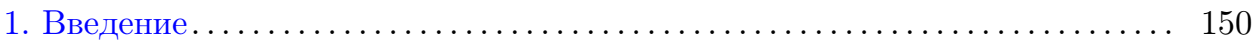

2. Глобальные по времени слабые решения ...................... 152

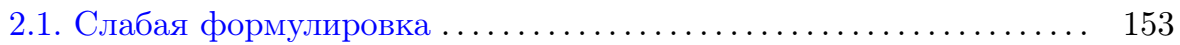

2.2. Определяющие уравнения . . . . . . . . . . . . . . . . 154

2.3. Глобальные по времени слабые решения системы Навье-Сток-

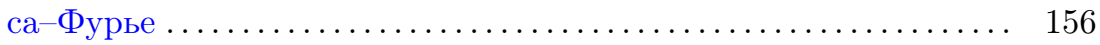

3. Корректность и устойчивость .......................... 159

3.1. Относительная энтропия и диссипативные решения.......... 159

3.2. Единственность сильных решений в классе слабых............ 161

3.3. Условная регулярность .............................. 161

3.4. Невязкая жидкость ............................... 162

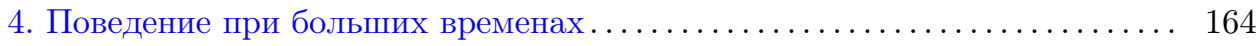



4.2. Глобальный аттрактор в потенциальном случае ............ 166

4.3. Неконсервативные движущие силы ................. 167

4.4. Системы, на которые действуют осциллирующие силы ........ 169

Работа выполнена при поддержке Европейского Совета по научным исследованиям в рамках Седьмой рамочной программы EC (FP7/2007-2013), грант ERC 320078.

(C) Э. ФАЙРАЙЗЛ, 2014 
5. Примеры поведения при больших временах для неконсервативных сис-

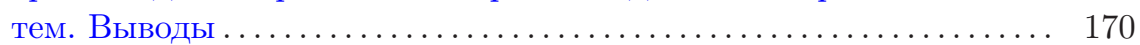

5.1. Периодическое по времени движение .................. 170

5.2. Заключительные комментарии . . . . . . . . . . . . . . . . 172



\section{1. Введение}

Многие важные особенности динамики жидкостей и, в частности, явления, связанные с турбулентностъю, происходят при больших значениях времени. Поведение при больших временах бесконечномерных динамических систем, порожденных уравнениями движущихся жидкостей, является одним из самых изученных разделов математической теории динамики жидкостей - по этому вопросу мы отсылаем читателя к ставшим классическими трудам Бабина и Вишика [3], Чепыжова и Вишика [11], Константина и Фояша [13], Константина и др. [14], Константина, Фояша и Темама [15], Экманна и Рюэлля [21], Фояша и др. [34], Ладыженской [40], [42], Кинь [48], Темама [53] и др.

В соответствии с фундаментальными принципами классической механики сплошной среды эволюция жидкости во времени описывается в терминах трех переменных состояния:

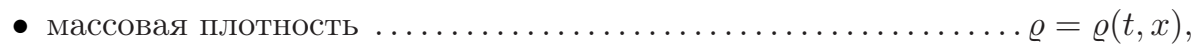

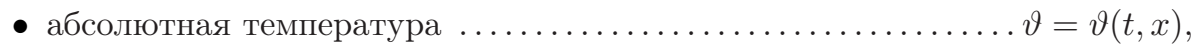

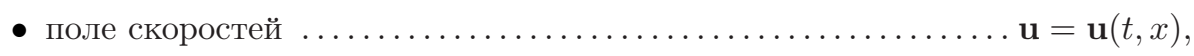

при этом все данные величины, являющиеся функциями времени $t$ и пространственного положения $x$, принадлежат физической отсчетной области $\Omega \subset \mathbb{R}^{3}$.

В эйлеровой системе координат, связанной с физическим пространством, а не с линиями тока, движение жидкости описывается уравнениями поля:

$$
\begin{aligned}
& \text { УРАВНЕНИЕ СОХРАНЕНИЯ МАССЫ } \\
& \partial_{t} \varrho+\operatorname{div}_{x}(\varrho \mathbf{u})=0 \\
& \text { УРАВНЕНИЕ СОХРАНЕНИЯ ИМПУЛЬСА } \\
& \partial_{t}(\varrho \mathbf{u})+\operatorname{div}_{x}(\varrho \mathbf{u} \otimes \mathbf{u})+\nabla_{x} p=\operatorname{div}_{x} \mathbb{S}+\varrho \mathbf{f} ; \\
& \text { УРАВНЕНИЕ СОХРАНЕНИЯ ВНУТРЕННЕЙ ЭНЕРГИИ } \\
& \partial_{t}(\varrho e)+\operatorname{div}_{x}(\varrho e \mathbf{u})+\operatorname{div}_{x} \mathbf{q}=\mathbb{S}: \nabla_{x} \mathbf{u}-p \operatorname{div}_{x} \mathbf{u}
\end{aligned}
$$

В (1.1)-(1.3) также участвуют следующие величины:

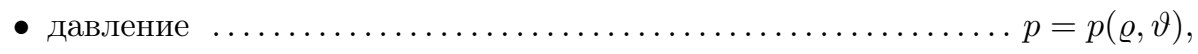

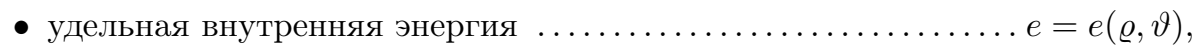

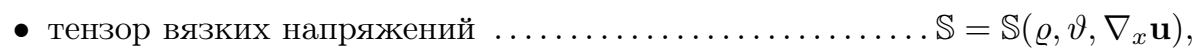


- внешняя объемная сила $\ldots \ldots \ldots \ldots \ldots \ldots \ldots \ldots \ldots \ldots \ldots \ldots \ldots \ldots . \mathbf{f}=\mathbf{f}(t, x)$,

• поток внутренней энергии $\left.\ldots \ldots \ldots \ldots \ldots \ldots \ldots \ldots \ldots . . \ldots \ldots \ldots \ldots, q \ldots, \nabla_{x} \vartheta\right)$.

Ниже, если не оговорено противное, мы будем рассматривать жидкости, заключенные в ограниченном физическом пространстве $\Omega \subset \mathbb{R}^{3}$, граница которого механически и теплоизолирована. Конечно, граничные условия, удовлетворяющие этому требованию, можно выбирать по-разному - мы рассмотрим следующие.

\section{НЕПРОНИЦАЕМОСТЬ$$
\left.\mathbf{u} \cdot \mathbf{n}\right|_{\partial \Omega}=0
$$

где $\mathbf{n}$ - внешний нормальный вектор;

УСЛОВИЕ ПРИЛИПАНИЯ или ПОЛНОЕ ПРОСКАЛЬЗЫВАНИЕ

$$
\left.\mathbf{u}_{\mathrm{tg}}\right|_{\partial \Omega}=0 \quad \text { или } \quad[\mathbb{S} \cdot \mathbf{n}]_{\mathrm{tg}}=0 ;
$$

ОТСУТСТВИЕ ВНУТРЕННЕГО ПОТОКА ЭНЕРГИИ

$$
\left.\mathbf{q} \cdot \mathbf{n}\right|_{\partial \Omega}=0
$$

Начальное состояние жидкой системы описывается стандартными

\section{НАЧАЛЬНЫМИ УСЛОВИЯМИ}

$$
\varrho(0, \cdot)=\varrho_{0}, \quad \vartheta(0, \cdot)=\vartheta_{0}, \quad \mathbf{u}(0, \cdot)=\mathbf{u}_{0}
$$

ПРимечАниЕ 1.1. Строго говоря, принимая во внимание специфический вид уравнений поля (1.1)-(1.3), начальные условия должны быть заданы в терминах $\varrho(0, \cdot),(\varrho \mathbf{u})(0, \cdot)$ и $\varrho e(\varrho, \vartheta)(0, \cdot)$. Однако нам представляется более удобным использовать для этой цели исходные переменные состояния.

В данной работе мы рассматриваем задачу (1.1)-(1.7) в рамках общей теории бесконечномерных динамических систем. В этой связи нам потребуются некоторые предварительные сведения о корректности и устойчивости глобальных по времени решений. В частности, мы рассмотрим следующие вопросы.

1. Физически допустимые определяющие уравнения и глобальное по времени существование (раздел 2). При исследовании систем нелинейных законов сохранения типа (1.1)-(1.3) возникает известная проблема отсутствия достаточно сильных априорных оценок, гарантирующих, что все члены в слабой формулировке по крайней мере равностепенно интегрируемы. Мы определяем класс физически допустимых определяющих уравнений, а также даем подходящую слабую формулировку, в которой задача (1.1)-(1.7) имеет 
по крайней мере одно слабое решение при любом выборе начальных данных, обладающих конечной энергией.

2. Корректность и устойчивость (раздел 3). В целом вопрос корректности задачи (1.1)-(1.7) остается по существу открытым, за исключением хорошо изученного случая гладких или малых начальных данных. Мы вводим понятие диссипативного решения, основываясь на так называемом неравенстве для относительной энтропии (энергии). Мы показываем, что диссипативные решения удовлетворяют принципу единственности сильных решений в классе слабых, что означает, что диссипативное решение совпадает с (гипотетическим) сильным решением с такими же начальными данными на максимальном интервале существования последнего. Мы коротко останавливаемся на теории гиперболических (невязких) систем, для которых метод выпуклого интегрирования, недавно разработанный ДеЛеллисом и Секелихиди [18] для задач несжимаемых систем Эйлера, дает бесконечно много слабых решений для специального класса начальных данных.

3. Второй закон термодинамики и асимптотическое поведение (раздел 4). Закончив изложение предварительных сведений, мы перейдем к рассмотрению задачи поведения при больших временах глобальных по времени слабых решений. Оказывается, что второй закон термодинамики, реализующийся в системе через эффективную вязкость и теплопроводность, имеет большое влияние на асимптотические свойства решений. Под действием консервативной силы $\mathbf{f}=\nabla_{x} F(x)$, действующей на систему, все решения стремятся к единственному равновесному решению, которое можно определить по начальным данным. В таком случае множество равновесных решений представляет собой (неограниченный) аттрактор задачи (1.1)-(1.7). С другой стороны, если $\mathbf{f} \neq \nabla_{x} F$, то траектории становятся неограниченными при $t \rightarrow \infty$.

4. Специальные вопросы и перспективы (раздел 5). В завершающей части работы обсуждаются некоторые частные случаи, которые включают в себя системы, на которые действуют периодические по времени внешние силы. В заключение дается небольшой обзор по дальнейшим перспективным исследованиям.

\section{2. Глобальные по времени слабые решения}

Вслед за пионерской работой Лере [43] вопрос существования глобальных по времени регулярных решений для задач механики жидкости, основанных на системах Навье-Стокса, рассматривался в многочисленных работах, как теоретических, так и ориентированных на практическое примерение (Антонцев, Кажихов, Монахов [2], Каффарелли, Кон, Ниренберг [8], Като [39], Ладыженская [40], [41], Лионс [44], [45], Темам [52] и многие другие). Задача по существу остается нерешенной - даже в "простом" несжимаемом случае в естественной трехмерной топологии нет ни положительных результатов для больших наборов данных, ни контрпримеров (см. работы Феффермана [25] и Тао [51]). Исходя из неявной предпосылки, что для более сложной системы (1.1)-(1.7) задача становится еще более деликатной, мы ограничимся классом слабых решений. 
2.1. Слабая формулировка. Для начала мы сформулируем общие предположения о термодинамических функциях $p=p(\varrho, \vartheta), e=e(\varrho, \vartheta)$. Дело в том, что $p$ и $e$ могут быть представлены как функции от термостатических переменных $\varrho, \vartheta$ при общепринятом предположении, что на временных шкалах, соответствующих макроскопическим моделям, в каждый момент времени жидкости находятся в состоянии термодинамического равновесия (Галлавотти [35], [36]).

Как следствие, в оставшейся части работы мы предполагаем выполненными следующие условия.

$$
\begin{gathered}
\text { СоотношЕниЕ ГиББСА } \\
\vartheta D s(\varrho, \vartheta)=D e(\varrho, \vartheta)+p(\varrho, \vartheta) D\left(\frac{1}{\varrho}\right), \\
\text { где } s=s(\varrho, \vartheta)-\text { удельная энтропия, } \\
\text { УСловиЕ ТЕРмодинАмичЕСКой УСТойчивости } \\
\frac{\partial p(\varrho, \vartheta)}{\partial \varrho}>0, \quad \frac{\partial e(\varrho, \vartheta)}{\partial \vartheta}>0 .
\end{gathered}
$$

2.1.1. Уравнение энтропии и уравнение полной энергии. В соответствии с соотношением Гиббса (2.1) уравнение сохранения внутренней энергии (1.3) может быть записано в форме уравнения для производства энтропии

$$
\partial_{t}(\varrho s(\varrho, \vartheta))+\operatorname{div}_{x}(\varrho s(\varrho, \vartheta) \mathbf{u})+\operatorname{div}_{x}\left(\frac{\mathbf{q}}{\vartheta}\right)=\sigma,
$$

где скорость производства энтропии равна

$$
\sigma=\frac{1}{\vartheta}\left(\mathbb{S}: \nabla_{x} \mathbf{u}-\frac{\mathbf{q} \cdot \nabla_{x} \vartheta}{\vartheta}\right) .
$$

Далее, умножая уравнение сохранение импульса (1.2) на и и складывая результат умножения с уравнением сохранения внутренней энергии (1.3), мы получаем уравнение полной энергии в виде

$$
\begin{gathered}
\partial_{t}\left(\frac{1}{2} \varrho|\mathbf{u}|^{2}+\varrho e(\varrho, \vartheta)\right)+\operatorname{div}_{x}\left[\left(\frac{1}{2} \varrho|\mathbf{u}|^{2}+\varrho e(\varrho, \vartheta)\right) \mathbf{u}\right] \\
+\operatorname{div}_{x}(p(\varrho) \mathbf{u}+\mathbf{q})-\operatorname{div}_{x}(\mathbb{S} \cdot \mathbf{u})=\varrho \mathbf{f} \cdot \mathbf{u} .
\end{gathered}
$$

Следует отметить, что уравнения (1.3), (2.3), (2.5) эквивалентны при условии, что все величины в (1.1)-(1.3) являются гладкими. Интегрируя (2.5) по физической области $\Omega$ и учитывая граничные условия (1.4)-(1.6), мы имеем

$$
\frac{\mathrm{d}}{\mathrm{d} t} \int_{\Omega}\left(\frac{1}{2} \varrho|\mathbf{u}|^{2}+\varrho e(\varrho, \vartheta)\right) \mathrm{d} x=\int_{\Omega} \varrho \mathbf{f} \cdot \mathbf{u} \mathrm{d} x .
$$

Таким образом, если $\mathbf{f}=0$, то полная энергия системы сохраняется. 
2.1.2. Неравенство для энтропии и слабая формулировка. Рассмотрим слабую формулировку задачи (1.1)-(1.3), в которую входят:

- уравнения (1.1), (1.2), понимаемые в смысле обобщенных функций, при этом уравнение сохранения внутренней энергии (1.3) заменяется на

УРАВНЕНИЕ ДЛЯ ПРОИЗВОДСТВА ЭНТРОПИИ

$$
\begin{gathered}
\partial_{t}(\varrho s(\varrho, \vartheta))+\operatorname{div}_{x}(\varrho s(\varrho, \vartheta) \mathbf{u})+\operatorname{div}_{x}\left(\frac{\mathbf{q}}{\vartheta}\right)=\sigma, \\
\text { где } \\
\sigma \geqslant \frac{1}{\vartheta}\left(\mathbb{S}: \nabla_{x} \mathbf{u}-\frac{\mathbf{q} \cdot \nabla_{x} \vartheta}{\vartheta}\right) \\
\text { дополненное }
\end{gathered}
$$

УРАВНЕНИЕМ ПОЛНОЙ ЭНЕРГИИ

$$
\frac{\mathrm{d}}{\mathrm{d} t} \int_{\Omega}\left(\frac{1}{2} \varrho|\mathbf{u}|^{2}+\varrho e(\varrho, \vartheta)\right) \mathrm{d} x=\int_{\Omega} \varrho \mathbf{f} \cdot \mathbf{u} \mathrm{d} x
$$

Аналогично (2.1), (2.2) соотношения (2.7)-(2.9) должны пониматься в смысле обобщенных функций. При замене (2.4) на неравенство мы допускаем появление возможных сингулярностей у слабых решений. Уравнение полной энергии (2.9) добавлено в систему для компенсации потерь информации из-за неравенства (2.8). Можно показать (см. [30; гл. 3]), что (2.7), (2.8) приводят к начальному уравнению сохранения внутренней энергии (1.3) при условии, что все величины гладкие. Мы увидим ниже, что формулировка, основанная на выражении для производства энтропии и уравнении полной энергии, достаточно удобна, поскольку она уже включает в себя так называемое относительное неравенство для энтропии, которое является основным инструментом во многих задачах об асимптотическом поведении систем.

2.2. Определяющие уравнения. Наш выбор определяющих уравнений частично обусловлен нуждами математической теории. При таком выборе достигается компромисс между физической сутью и необходимыми априорными оценками, получаемыми через определяющие уравнения. По поводу дальнейших деталей мы отсылаем читателя к главе 1 монографии [30].

2.2.1. Термодинамические функции. Как мы уже отметили в п. 2.1, термодинамические величины $p, e, s$ явно зависят от термостатических переменных $\varrho, \vartheta$, связанных между собой через соотношения Гиббса (2.1) и удовлетворяющих условию термодинамической устойчивости (2.2). Дополнительно мы вводим уравнение состояния, являющееся типичным для молекулярного давления $p_{M}$ одноатомных газов. Именно,

$$
p_{M}(\varrho, \vartheta)=\frac{2}{3} \varrho e_{M}(\varrho, \vartheta) .
$$


Отсюда с учетом (2.1) мы получаем универсальную формулу

$$
p_{M}(\varrho, \vartheta)=\vartheta^{5 / 2} P\left(\frac{\varrho}{\vartheta^{3 / 2}}\right)
$$

для некоторой функции $P$. Если функция $P$ линейна, то мы немедленно получаем уравнение идеального газа $p_{M}=a \varrho \vartheta$.

В соответствии с (2.10) имеем

$$
e_{M}(\varrho, \vartheta)=\frac{3}{2} \vartheta\left(\frac{\vartheta^{3 / 2}}{\varrho}\right) P\left(\frac{\varrho}{\vartheta^{3 / 2}}\right)
$$

и, возвращаясь обратно к (2.1),

$$
s_{M}(\varrho, \vartheta)=S\left(\frac{\varrho}{\vartheta^{3 / 2}}\right), \quad \text { где } \quad S^{\prime}(Z)=-\frac{3}{2} \frac{(5 / 3) P(Z)-P^{\prime}(Z) Z}{Z^{2}} .
$$

Более того, из условия термодинамической устойчивости (2.2) мы получаем следующие ограничения на $P$ :

- так как $\partial_{\varrho} p>0$, то $P^{\prime}(Z)>0$;

- так как $\partial_{\vartheta} e>0$, то

$$
\frac{(5 / 3) P(Z)-P^{\prime}(Z) Z}{Z}>0
$$

- функция $Z \mapsto P(Z) / Z^{5 / 3}$ убывает в силу (2.14); соответственно, мы предполагаем, что

$$
\lim _{Z \rightarrow \infty} \frac{P(Z)}{Z^{5 / 3}}=p_{\infty}>0
$$

- функция $Z \mapsto S(Z)$ убывает в силу (2.13); мы предполагаем, что

$$
\lim _{Z \rightarrow \infty} S(Z)=0
$$

что согласуется с третьим законом термодинамики (см. работы Бельджорно [5], [6] и Кэллена [9]).

2.2.2. Тепловое излучение. Наличие эффекта теплового излучения требует модификации давления, а также других термодинамических функций путем введения "излучательных" компонент. Этого можно добиться, положив

$$
\begin{aligned}
& p(\varrho, \vartheta)=p_{M}(\varrho, \vartheta)+\frac{a}{3} \vartheta^{4}, \quad a>0, \\
& e(\varrho, \vartheta)=e_{M}(\varrho, \vartheta)+\frac{a}{\varrho} \vartheta^{4}
\end{aligned}
$$

и, с учетом соотношения Гиббса (2.1),

$$
s(\varrho, \vartheta)=s_{M}(\varrho, \vartheta)+\frac{4 a}{3} \frac{\vartheta^{3}}{\varrho} .
$$

По поводу других возможных форм уравнений состояния, а также детального обсуждения и физических основ мы отсылаем читателя к монографии [22]. 
2.2.3. Коэффициенты переноса. Мы рассматриваем нъютоновские жидкоcmu, для которых для вязких напряжений выполняется реологическое уравнение Ньютона

$$
\mathbb{S}\left(\vartheta, \nabla_{x} \mathbf{u}\right)=\mu(\vartheta)\left(\nabla_{x} \mathbf{u}+\nabla_{x}^{t} \mathbf{u}-\frac{2}{3} \operatorname{div}_{x} \mathbf{u I}\right)+\eta(\vartheta) \operatorname{div}_{x} \mathbf{u I}
$$

где коэффициент сдвиговой вязкости $\mu$ и коэффициент объемной вязкости $\eta$ могут зависеть от термостатических переменных $\varrho, \vartheta$. Из технических соображений мы можем рассмотреть лишь случай $\mu=\mu(\vartheta), \eta=\eta(\vartheta)$. K примеру, такое условие возникает в случае газов (см. работу Беккера [4]).

Аналогично, тепловой поток определяется

$$
\begin{gathered}
\text { ЗАКоном Фурье } \\
\mathbf{q}\left(\vartheta, \nabla_{x} \vartheta\right)=-\kappa(\vartheta) \nabla_{x} \vartheta .
\end{gathered}
$$

Уравнения поля (1.1)-(1.3), где $\mathbb{S}$ и q определяются из $(2.20),(2.21)$, называются системой Навъе-Стокса-Фуръе.

\section{3. Глобальные по времени слабые решения системы Навье-Сток-} са-Фурье. Мы говорим, что тройка $[\varrho, \vartheta, \mathbf{u}]$ является слабым решением системы Навье-Стокса- Фурье в пространственно-временном цилиндре $(0, T) \times \Omega$, если:

- плотность и температура положительны, $\varrho(t, x) \geqslant 0, \vartheta(t, x)>0$ для почти всех $(t, x) \in(0, T) \times \Omega$, а также

$$
\begin{gathered}
\varrho \in C_{\text {weak }}\left([0, T] ; L^{5 / 3}(\Omega)\right), \quad(\varrho \mathbf{u}) \in C_{\text {weak }}\left([0, T] ; L^{\beta}\left(\Omega ; \mathbb{R}^{3}\right)\right), \\
\vartheta \in L^{\infty}\left(0, T ; L^{s}(\Omega)\right) \cap L^{2}\left(0, T ; W^{1,2}(\Omega)\right), \\
\mathbf{u} \in L^{2}\left(0, T ; W^{1, r}\left(\Omega ; \mathbb{R}^{3}\right)\right) \quad \text { при некоторых } r, s, \beta>1, \\
\left.\mathbf{u}\right|_{\partial \Omega}=0 \quad \text { или }\left.\quad \mathbf{u} \cdot \mathbf{n}\right|_{\partial \Omega}=0 ;
\end{gathered}
$$

- уравнение непрерывности (1.1) заменяется на

$$
\begin{aligned}
\int_{\Omega}(\varrho & +b(\varrho))(\tau, \cdot) \varphi(\tau, \cdot) \mathrm{d} x-\int_{\Omega}\left(\varrho_{0}+b\left(\varrho_{0}\right)\right) \varphi(0, \cdot) \mathrm{d} x \\
& =\int_{0}^{\tau} \int_{\Omega}\left[(\varrho+b(\varrho)) \partial_{t} \varphi+(\varrho+b(\varrho)) \mathbf{u} \cdot \nabla_{x} \varphi+\left(b(\varrho)-b^{\prime}(\varrho) \varrho\right) \operatorname{div}_{x} \mathbf{u} \varphi\right] \mathrm{d} x \mathrm{~d} t
\end{aligned}
$$

для любой основной функции $\varphi \in C^{1}([0, T] \times \bar{\Omega}), \tau \in[0, T]$, и любого $b \in$ $C^{1}[0, \infty), b(0)=0, b^{\prime}(\varrho)=0$ при всех $\varrho \geqslant c_{b}$; 
- уравнение движения (1.2) выполнено в смысле обобщенных функций

$$
\begin{aligned}
& \int_{\Omega} \varrho \mathbf{u}(\tau, \cdot) \cdot \varphi(\tau, \cdot) \mathrm{d} x-\int_{\Omega} \varrho_{0} \mathbf{u}_{0} \cdot \varphi(0, \cdot) \mathrm{d} x \\
& \quad=\int_{0}^{\tau} \int_{\Omega}\left(\varrho \mathbf{u} \cdot \partial_{t} \varphi+\varrho \mathbf{u} \otimes \mathbf{u}: \nabla_{x} \varphi+p(\varrho, \vartheta) \operatorname{div}_{x} \varphi-\mathbb{S}: \nabla_{x} \varphi+\varrho \mathbf{f} \cdot \varphi\right) \mathrm{d} x \mathrm{~d} t
\end{aligned}
$$

для любой $\varphi \in C^{1}\left([0, T] \times \bar{\Omega} ; \mathbb{R}^{3}\right)$, где $\left.\varphi\right|_{\partial \Omega}=0$ в случае граничных условий прилипания и $\left.\varphi \cdot \mathbf{n}\right|_{\partial \Omega}=0$ в случае граничных условий полного проскальзывания;

• уравнение баланса энтропии (2.7), (2.8) заменяется на семейство интегральных неравенств

$$
\begin{aligned}
\int_{\Omega} \varrho_{0} s\left(\varrho_{0}, \vartheta_{0}\right) \varphi(0, \cdot) \mathrm{d} x-\int_{\Omega} \varrho s(\varrho, \vartheta)(\tau, \cdot) \varphi(\tau, \cdot) \mathrm{d} x \\
\quad+\int_{0}^{\tau} \int_{\Omega} \frac{\varphi}{\vartheta}\left(\mathbb{S}: \nabla_{x} \mathbf{u}-\frac{\mathbf{q} \cdot \nabla_{x} \vartheta}{\vartheta}\right) \mathrm{d} x \mathrm{~d} t \\
\leqslant-\int_{0}^{\tau} \int_{\Omega}\left(\varrho s(\varrho, \vartheta) \partial_{t} \varphi+\varrho s(\varrho, \vartheta) \mathbf{u} \cdot \nabla_{x} \varphi+\frac{\mathbf{q} \cdot \nabla_{x} \varphi}{\vartheta}\right) \mathrm{d} x \mathrm{~d} t
\end{aligned}
$$

для любой $\varphi \in C^{1}([0, T] \times \bar{\Omega}), \varphi \geqslant 0$, и почти всех $\tau \in[0, T]$;

- полная энергия удовлетворяет уравнению

$$
\begin{aligned}
& \int_{\Omega}\left(\frac{1}{2} \varrho|\mathbf{u}|^{2}+\varrho e(\varrho, \vartheta)\right)(\tau, \cdot) \mathrm{d} x \\
& \quad=\int_{\Omega}\left(\frac{1}{2} \varrho_{0}\left|\mathbf{u}_{0}\right|^{2}+\varrho_{0} e\left(\varrho_{0}, \vartheta_{0}\right)\right) \mathrm{d} x+\int_{0}^{\tau} \int_{\Omega} \varrho \mathbf{f} \cdot \mathbf{u} \mathrm{d} x \mathrm{~d} t
\end{aligned}
$$

для почти всех $\tau \in[0, T]$.

ПримечАниЕ 2.1. Интегральное соотношение (2.22) соответствует так называемому перенормированному варианту уравнения непрерывности (1.1), введенному ДиПерна и Лионсом [20]. Отметим, что (2.22) сводится к стандартной слабой формулировке (1.1), если $b=0$.

Как показано в [30; гл. 2], слабое решение является сильным и, в частности, уравнение баланса энтропии удовлетворяется со знаком равенства при условии, что все величины, участвующие в слабой формулировке, являются достаточно гладкими.

Предположения и основная теорема существования. В соответствии со сказанным выше мы предположим, что функции $p, e, s, \mu, \eta, \kappa$ удовлетворяют следующим предположениям.

1. Функции $p, e, s$ определены в (2.17)-(2.19), где

$$
\begin{gathered}
P \in C^{1}[0, \infty) \cap C^{3}(0, \infty), \quad P(0)=0, \\
P^{\prime}(Z)>0, \quad 0<\frac{(5 / 3) P(Z)-P^{\prime}(Z) Z}{Z}<c \quad \text { для всех } Z>0, \\
\lim _{Z \rightarrow \infty} \frac{P(Z)}{Z^{5 / 3}}=p_{\infty}>0, \quad \lim _{Z \rightarrow \infty} S(Z)=0 .
\end{gathered}
$$


2. Коэффициенты вязкости являются непрерывно дифференцируемыми функциями от температуры такими, что

$$
\begin{gathered}
0<\underline{\mu}\left(1+\vartheta^{\Lambda}\right) \leqslant \mu(\vartheta) \leqslant \bar{\mu}\left(1+\vartheta^{\Lambda}\right), \quad\left|\mu^{\prime}(\vartheta)\right|<c \\
\text { для всех } \vartheta \in[0, \infty) \text { и } \frac{2}{5}<\Lambda \leqslant 1, \\
0 \leqslant \eta(\vartheta) \leqslant \bar{\eta}\left(1+\vartheta^{\Lambda}\right) \quad \text { для всех } \vartheta \in[0, \infty) .
\end{gathered}
$$

3. Коэффициент теплопроводности $\kappa=\kappa(\vartheta)$ удовлетворяет неравенству

$$
0<\underline{\kappa}\left(1+\vartheta^{3}\right) \leqslant \kappa(\vartheta) \leqslant \bar{\kappa}\left(1+\vartheta^{3}\right) \text { для всех } \vartheta \in[0, \infty) .
$$

Отметим следующую теорему существования [30; гл. 3, теорема 3.1].

ТЕОрема 2.1. Пусть $\Omega \subset \mathbb{R}^{3}$ - ограниченная область с границей класса $C^{2+\nu}$. Предположим, что термодинамические функции $p, e, s$ и коэфбициенты переноса $\mu, \eta, \kappa$ удовлетворяют предположениям 1-3, сформулированным выше. Далее, предположим, что начальные данные таковы, что

$$
\begin{aligned}
& \varrho_{0} \in L^{5 / 3}(\Omega), \quad \vartheta_{0} \in L^{\infty}(\Omega), \quad \varrho_{0}, \vartheta_{0}>0, \\
& \mathbf{u}_{0} \in W^{1, \infty}\left(\Omega ; \mathbb{R}^{3}\right), \quad \mathbf{f} \in L^{\infty}\left((0, T) \times \Omega ; \mathbb{R}^{3}\right) .
\end{aligned}
$$

Тогда система Навъе-Стокса-Фуръе (2.22)-(2.25) имеет слабое решение $[\varrho, \vartheta, \mathbf{u}]$ на произвольном временном интервале $(0, T)$. При этом слабое решение обладает следующими свойствами:

$\varrho \geqslant 0$ n.в. в $(0, T) \times \Omega, \quad \varrho \in C\left([0, T] ; L^{1}(\Omega)\right) \cap L^{\infty}\left(0, T ; L^{5 / 3}(\Omega)\right) \cap L^{\delta}((0, T) \times \Omega)$

при некотором $\delta>5 / 3$; более того,

$$
\begin{gathered}
\vartheta>0 \text { п.в. в }(0, T) \times \Omega, \quad \vartheta \in L^{\infty}\left(0, T ; L^{4}(\Omega)\right) \cap L^{2}\left(0, T ; W^{1,2}(\Omega)\right), \\
\vartheta^{3}, \log (\vartheta) \in L^{2}\left(0, T ; W^{1,2}(\Omega)\right) ; \\
\mathbf{u} \in L^{2}\left(0, T ; W_{0}^{\alpha}\left(\Omega ; \mathbb{R}^{3}\right)\right), \quad \alpha=\frac{8}{5-\Lambda}, \quad \varrho \mathbf{u} \in C_{\text {weak }}\left(0, T ; L^{5 / 4}\left(\Omega ; \mathbb{R}^{3}\right)\right) .
\end{gathered}
$$

ПримечАниЕ 2.2. В теории динамических систем более удобно работать с глобальными по времени решениями. По определению, $[\varrho, \vartheta, \mathbf{u}]$ есть слабое решение системы Навье-Стокса-Фурье на $[0, \infty) \times \Omega$, если уравнения $(2.22)-(2.25)$ выполнены на любом конечном интервале $[0, T], T>0$. Теорема существования (теорема 2.1) также остается верной на $[0, \infty)$.

Предположения теоремы 2.1 не являются оптимальными. По поводу возможных обобщений мы отсылаем читателя к [30; гл. 3].

Полное доказательство теоремы 2.1 довольно длинное и носит технический характер. Основными составляющими доказательства являются следующие:

- общая теория существования для сжимаемых баротропных систем Навье-Стокса, разработанная Лионсом [45]; в частности, соотношение из [45] удовлетворяется для эффективного вязкого давления;

- понятие меры дефекта осцилляций, введенное в [26];

• развитие теории на случай переменных коэффициентов переноса [27]. 


\section{3. Корректность и устойчивость}

Коротко остановимся на вопросе корректности и устойчивости системы Навье-Стокса-Фурье для слабых решений. Для этой цели мы введем термодинамический потенциал, называемый баллистической энергией:

$$
H_{\Theta}(\varrho, \vartheta)=\varrho(e(\varrho, \vartheta)-\Theta s(\varrho, \vartheta))
$$

(см. работу Эриксена [23]).

Следующие два свойства потенциала $H_{\Theta}$, играющие ключевую роль в изучении устойчивости, непосредственно вытекают из предположения о термодинамической устойчивости (2.2):

- функция $\varrho \mapsto H_{\Theta}(\varrho, \Theta)$ строго выпукла по $\varrho$;

- функция $\vartheta \mapsto H_{\Theta}(\varrho, \vartheta)$ убывает при $\vartheta<\Theta$ и возрастает при $\vartheta>\Theta$ для любого $\varrho$.

3.1. Относительная энтропия и диссипативные решения. Отталкиваясь от исследований Дафермоса [16] и Джермейна [37], мы введем функционал относительной энтропии (энергии):

$\mathscr{E}(\varrho, \vartheta, \mathbf{u} \mid r, \Theta, \mathbf{U})=\int_{\Omega}\left(\frac{1}{2} \varrho|\mathbf{u}-\mathbf{U}|^{2}+H_{\Theta}(\varrho, \vartheta)-\frac{\partial H_{\Theta}(r, \Theta)}{\partial \varrho}(\varrho-r)-H_{\Theta}(r, \Theta)\right) \mathrm{d} x$.

С учетом уже упомянутых свойств функции $H_{\Theta}$ отметим, что функция $\mathscr{E}$ является (несимметричным) расстоянием между тройками $[\varrho, \vartheta, \mathbf{u}]$ и $[r, \Theta, \mathbf{U}]$.

3.1.1. Неравенство для относительной энтропии. Записывая относительную энтропию в виде

$$
\mathscr{E}(\varrho, \vartheta, \mathbf{u} \mid r, \Theta, \mathbf{U})=\sum_{j=1}^{5} I_{j}
$$

где

$$
\begin{aligned}
& I_{1}=\int_{\Omega}\left(\frac{1}{2} \varrho|\mathbf{u}|^{2}+\varrho e(\varrho, \vartheta)\right) \mathrm{d} x, \\
& I_{2}=-\int_{\Omega} \varrho \mathbf{u} \cdot \mathbf{U} \mathrm{d} x, \\
& I_{3}=\int_{\Omega} \varrho\left(\frac{1}{2}|\mathbf{U}|^{2}-\frac{\partial H_{\Theta}(r, \Theta)}{\partial \varrho}\right) \mathrm{d} x, \\
& I_{4}=-\int_{\Omega} \Theta \varrho s(\varrho, \vartheta) \mathrm{d} x, \\
& I_{5}=\int_{\Omega}\left(\frac{\partial H_{\Theta}(r, \Theta)}{\partial \varrho} r+H_{\Theta}(r, \Theta)\right) \mathrm{d} x,
\end{aligned}
$$

мы видим, что $\mathscr{E}$ может использоваться для оценки изменения со временем расстояния между слабым решением $[\varrho, \vartheta, \mathbf{u}]$ и произвольной тройкой гладких 
функций $[r, \Theta, \mathbf{U}]$, удовлетворяющих естественным ограничениям

$$
r>0, \quad \Theta>0,
$$

$\left.\mathbf{U}\right|_{\partial \Omega}=0$, если $\mathbf{u}$ удовлетворяет граничному условию прилипания,

$\left.\mathbf{U} \cdot \mathbf{n}\right|_{\partial \Omega}=0$ в случае полного проскальзывания.

В самом деле, выражая изменение со временем для каждого из интегралов $I_{1}-I_{5}$ через интегральные тождества (2.22)-(2.25), мы получаем

$$
\begin{aligned}
& \text { НЕРАВЕНСТВО ДЛЯ ОТНОСИТЕЛЬНОЙ ЭНТРОПИИ } \\
& {[\mathscr{E}(\varrho, \vartheta, \mathbf{u} \mid r, \Theta, \mathbf{U})]_{t=0}^{t=\tau}} \\
& +\int_{0}^{\tau} \int_{\Omega} \frac{\Theta}{\vartheta}\left(\mathbb{S}\left(\vartheta, \nabla_{x} \mathbf{u}\right): \nabla_{x} \mathbf{u}-\frac{\mathbf{q}\left(\vartheta, \nabla_{x} \vartheta\right) \cdot \nabla_{x} \vartheta}{\vartheta}\right) \mathrm{d} x \mathrm{~d} t \\
& \leqslant \int_{0}^{\tau} \mathscr{R}(\varrho, \vartheta, \mathbf{u}, r, \Theta, \mathbf{U}) \mathrm{d} t \quad \text { для почти всех } \tau \geqslant 0 \text {, }
\end{aligned}
$$

где остаток имеет вид

$$
\begin{aligned}
\mathscr{R}(\varrho, \vartheta, \mathbf{u}, & r, \Theta, \mathbf{U}) \\
=\int_{0}^{\tau} & \int_{\Omega} \varrho(\mathbf{u}-\mathbf{U}) \cdot \nabla_{x} \mathbf{U} \cdot(\mathbf{U}-\mathbf{u}) \mathrm{d} x \mathrm{~d} t \\
& +\int_{0}^{\tau} \int_{\Omega} \varrho(s(\varrho, \vartheta)-s(r, \Theta))(\mathbf{U}-\mathbf{u}) \cdot \nabla_{x} \Theta \mathrm{d} x \mathrm{~d} t \\
& +\int_{0}^{\tau} \int_{\Omega}\left(\varrho\left(\partial_{t} \mathbf{U}+\mathbf{U} \cdot \nabla_{x} \mathbf{U}\right) \cdot(\mathbf{U}-\mathbf{u})-p(\varrho, \vartheta) \operatorname{div}_{x} \mathbf{U}\right. \\
& \left.+\mathbb{S}\left(\vartheta, \nabla_{x} \mathbf{u}\right): \nabla_{x} \mathbf{U}\right) \mathrm{d} x \mathrm{~d} t \\
& -\int_{0}^{\tau} \int_{\Omega}\left(\varrho(s(\varrho, \vartheta)-s(r, \Theta)) \partial_{t} \Theta+\varrho(s(\varrho, \vartheta)-s(r, \Theta)) \mathbf{U} \cdot \nabla_{x} \Theta\right. \\
& \left.+\frac{\mathbf{q}\left(\vartheta, \nabla_{x} \vartheta\right)}{\vartheta} \cdot \nabla_{x} \Theta\right) \mathrm{d} x \mathrm{~d} t \\
& +\int_{0}^{\tau} \int_{\Omega}\left(\left(1-\frac{\varrho}{r}\right) \partial_{t} p(r, \Theta)-\frac{\varrho}{r} \mathbf{u} \cdot \nabla_{x} p(r, \Theta)\right) \mathrm{d} x \mathrm{~d} t \\
& +\int_{0}^{\tau} \int_{\Omega} \varrho \mathbf{f} \cdot(\mathbf{u}-\mathbf{U}) \mathrm{d} x \mathrm{~d} t
\end{aligned}
$$

(по поводу деталей см. [31]).

3.1.2. Диссипативные решения. Следуя монографии ДиПерна и Лионса [44], мы говорим, что $[\varrho, \vartheta, \mathbf{u}]$ является диссипативным решением системы НавьеСтокса-Фурье, если:

- $[\varrho, \vartheta, \mathbf{u}]$ принадлежит регулярному классу, задаваемому (2.32)-(2.35);

- $[\varrho, \vartheta, \mathbf{u}]$ удовлетворяет неравенству для относительной энтропии для почти всех $\tau \in[0, T]$ для любой тройки основных функций $[r, \Theta, \mathbf{U}]$, удовлетворяющих (3.3). 
Из предыдущего ясно, что любое слабое решение системы Навье-СтоксаФурье является диссипативным решением.

3.2. Единственность сильных решений в классе слабых. Примечательным свойством диссипативных решений является то, что для них выполняется принцип единственности силъных решений в классе слабых. Здесь мы уделим основное внимание случаю граничного условия прилипания для скорости. Рассмотрим регулярные начальные данные

$$
\begin{gathered}
\varrho(0, \cdot)=\varrho_{0}, \quad \inf _{\Omega} \varrho_{0}>0, \quad \varrho_{0} \in W^{3,2}(\Omega), \\
\vartheta(0, \cdot)=\vartheta_{0}, \quad \inf _{\Omega} \vartheta_{0}>0, \quad \vartheta_{0} \in W^{3,2}(\Omega), \\
\mathbf{u}(0, \cdot)=\mathbf{u}_{0} \in W^{3,2}\left(\Omega ; \mathbb{R}^{3}\right),
\end{gathered}
$$

удовлетворяющие естественным условиям совместности

$$
\begin{gathered}
\left.\mathbf{u}_{0}\right|_{\partial \Omega}=0,\left.\quad \nabla_{x} \vartheta_{0} \cdot \mathbf{n}\right|_{\partial \Omega}=0, \\
\left.\nabla_{x} p\left(\varrho_{0}, \vartheta_{0}\right)\right|_{\partial \Omega}=\operatorname{div}_{x} \mathbb{S}\left(\varrho_{0}, \vartheta_{0}, \nabla_{x} \mathbf{u}_{0}\right)+\left.\varrho_{0} \mathbf{f}\right|_{\partial \Omega} .
\end{gathered}
$$

Теорема 3.1. В условиях теоремы 2.1 предположим, что $[\varrho, \vartheta, \mathbf{u}]$ является диссипативным решением системы Навъе-Стокса-Фуръе с начальными данными из класса (3.6)-(3.9). Пусть

$$
\tilde{\varrho}, \tilde{\vartheta} \in C\left([0, T] ; W^{3,2}(\Omega)\right), \quad \tilde{\mathbf{u}} \in C\left([0, T] ; W^{3,2}\left(\Omega ; \mathbb{R}^{3}\right)\right)
$$

- сильное решение той же задачи, определенное на интервале существования $\left[0, T_{\max }\right), 0<T<T_{\max }$.

Тогда

$$
\varrho=\tilde{\varrho}, \quad \vartheta=\tilde{\vartheta}, \quad \mathbf{u}=\tilde{\mathbf{u}} \quad в[0, T] \times \Omega .
$$

Основная идея доказательства достаточно очевидна - мы берем $[\tilde{\varrho}, \tilde{\vartheta}, \tilde{\mathbf{u}}]$ в качестве основных функций в неравенстве для относительной энтропии (3.4). Тем не менее полное доказательство теоремы 3.1 получается длинным и скучным (см. [28; теорема 6.2] и [31; теорема 2.1]).

ПримечАниЕ 3.1. Существование локальных по времени сильных решений системы Навье-Стокса-Фурье было доказано Валли [54], [55] и Валли и Заяцковским [56]. Глобальное существование для "малых" начальных данных установлено Мацумурой и Нишидой [46], [47].

3.3. Условная регулярность. Другое приложение неравенства для относительной энтропии дается в следующем утверждении об условной регулярности (см. [32; теорема 2.1]).

Теорема 3.2. В условиях теоремы 2.1 предположим, что $[\varrho, \vartheta, \mathbf{u}]-\partial и с с и-$ пативное решение системы Навъе-Стокса-Фуръе в $(0, T) \times \Omega$ с началъными данными из класса (3.6)-(3.9), при этом

$$
\underset{(0, T) \times \Omega}{\operatorname{ess} \sup _{x}}\left|\nabla_{x} \mathbf{u}\right|<\infty .
$$

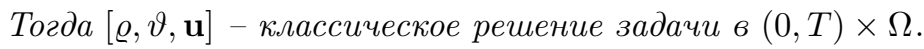


ПРимЕчАНИЕ 3.2. По поводу других результатов об условной регулярности см., например, работы Фана, Джана, Оу [24], Джана и Оу [38]. Основным преимуществом теоремы 3.2 является ее применимость в ситуации, когда известно, что слабые решения существуют глобально по времени.

3.4. Невязкая жидкость. На настоящий момент нет понимания, действительно ли необходимо использование слабых решений для задач с вязкими жидкостями. В отличие от ситуации с невязкими жидкостями, где ударные волны фактически всегда возникают вне зависимости от того, насколько гладкими или малыми являются начальные данные, аналогичные сингулярности не наблюдались для решений системы Навье-Стокса-Фурье. С другой стороны, задача о корректности и, в частности, о единственности в классе слабых решений остается по существу открытой даже для вязких жидкостей. Для исследования неожиданных свойств, которыми могут обладать слабые решения, нам потребуется провести небольшой экскурс в теорию невязких жидкостей, отталкиваясь от недавних результатов ДеЛеллиса и Секелихиди [17]-[19].

3.4.1. Система Эйлера-Фурье. Для системы (1.1)-(1.3) рассмотрим ее невязкий аналог, а именно, систему Эйлера-Фурье

$$
\begin{gathered}
\partial_{t} \varrho+\operatorname{div}_{x}(\varrho \mathbf{u})=0 \\
\partial_{t}(\varrho \mathbf{u})+\operatorname{div}_{x}(\varrho \mathbf{u} \otimes \mathbf{u})+\nabla_{x}(\varrho \vartheta)=0, \\
\frac{3}{2}\left(\partial_{t}(\varrho \vartheta)+\operatorname{div}_{x}(\varrho \vartheta \mathbf{u})\right)-\Delta \vartheta=-\varrho \vartheta \operatorname{div}_{x} \mathbf{u},
\end{gathered}
$$

где для простоты мы рассматриваем давление и внутреннюю энергию одноатомного газа. Несмотря на то что такая модель несколько непоследовательна (вязкость становится равной нулю, в то время как газ все еще теплопроводен), аналогичные уравнения использовались, например, в ряде приложений в метеорологии (см. работу Уилкокса [57]). Для того чтобы избежать рассмотрения технической задачи, связанной с присутствием кинематической границы, мы ограничимся случаем периодической пространственной области

$$
\Omega=\left(\left.[-1,1]\right|_{\{-1 ; 1\}}\right)^{3} .
$$

С математической точки зрения система (3.10)-(3.12) является гиперболопараболической и попадает в категорию задач, изученных Серром [49], [50]. В частности, задача имеет регулярные решения при регулярных начальных данных на возможно коротком временном отрезке (см. работы Алазара [1] и Серра [49]). Поскольку система (3.10)-(3.12) сходна по структуре со сжимаемой изэнтропической системой Эйлера, то вполне вероятно, что ударные волны будут возникать за конечное время даже для гладких и малых начальных данных.

3.4.2. Бесконечное число слабых решений для гладких начальных данных. Следующий достаточно неожиданный результат был получен модификацией метода комплексного интегрирования, разработанного ДеЛеллисом и Секелихиди [18] (см. [12; теорема 3.1]). 
Теорема 3.3. Пусть $T>0$, и пусть начальные данные таковы, что

$$
\begin{gathered}
\varrho_{0} \in C^{3}(\Omega), \quad \vartheta_{0} \in C^{2}(\Omega), \quad \mathbf{u}_{0} \in C^{3}\left(\Omega ; \mathbb{R}^{3}\right), \\
\varrho_{0}(x)>\underline{\varrho}>0, \quad \vartheta_{0}(x)>\underline{\vartheta}>0 \quad \text { для любого } x \in \Omega .
\end{gathered}
$$

Тогда задача Коши (3.10)-(3.13) допускает бесконечно много слабых решений в $(0, T) \times \Omega$ таких, что $\varrho \in C^{2}([0, T] \times \Omega)$,

$$
\begin{gathered}
\partial_{t} \vartheta \in L^{p}\left(0, T ; L^{p}(\Omega)\right), \quad \nabla_{x}^{2} \vartheta \in L^{p}\left(0, T ; L^{p}\left(\Omega ; \mathbb{R}^{3 \times 3}\right)\right) \quad \text { для любого } 1 \leqslant p<\infty, \\
\mathbf{u} \in C_{\text {weak }}\left([0, T] ; L^{2}\left(\Omega ; \mathbb{R}^{3}\right)\right) \cap L^{\infty}\left((0, T) \times \Omega ; \mathbb{R}^{3}\right), \quad \operatorname{div}_{x} \mathbf{u} \in C^{2}([0, T] \times \Omega) .
\end{gathered}
$$

ПРИмЕЧАниЕ 3.3. В предположении регулярности решение $[\varrho, \vartheta, \mathbf{u}]$ является "почти" сильным в смысле того, что для него в слабом смысле удовлетворяется лишь уравнение движения (3.11), в то время как (3.10), (3.12) выполняются поточечно в $(0, T) \times \Omega$.

Некоторые из полученных в теореме 3.3 решений, очевидно, являются "нефизическими". В частности, в точности так, как для системы Навье-Стокса-Фурье, можно показать, что слабые решения (3.10)-(3.14) удовлетворяют принципу единственности сильных решений в классе слабых. Фактически же они удовлетворяют неравенству для относительной энтропии (3.4) при $\mathbb{S}=0$ при условии, что для них сохраняется полная энергия, а именно,

$$
\begin{aligned}
& \int_{\Omega}(\left.\frac{1}{2} \varrho|\mathbf{u}|^{2}+\frac{3}{2} \varrho \vartheta\right)(\tau, \cdot) \mathrm{d} x \\
& \quad=\int_{\Omega}\left(\frac{1}{2} \varrho_{0}\left|\mathbf{u}_{0}\right|^{2}+\frac{3}{2} \varrho_{0} \vartheta_{0}\right) \mathrm{d} x \quad \text { для почти всех } \tau \in(0, T) .
\end{aligned}
$$

Таким образом, решения, полученные в теореме 3.3 , не удовлетворяют (3.15) в том смысле, что они "производят" энергию (или, в терминологии п. 3.1.2, не являются диссипативными).

С другой стороны, следующий результат показывает, что даже выполнение (3.15) не гарантирует единственности (см. [12; теорема 4.2]).

ТЕОРема 3.4. Пусть $T>0$, и пусть начальные данные $\varrho_{0}, \vartheta_{0}$ таковъ, что

$$
\varrho_{0}, \vartheta_{0} \in C^{2}(\Omega), \quad \varrho_{0}(x)>\underline{\varrho}>0, \quad \vartheta_{0}(x)>\underline{\vartheta}>0 \quad \text { для любого } x \in \Omega .
$$

Тогда существует поле скоростей $\mathbf{u}_{0}$,

$$
\mathbf{u}_{0} \in L^{\infty}\left(\Omega ; \mathbb{R}^{3}\right),
$$

такое, что задача (3.10)-(3.13) с начальными данными $\left[\varrho_{0}, \vartheta_{0}, \mathbf{u}_{0}\right]$ допускает бесконечное число слабых решений $(0, T) \times \Omega$, удовлетворяющих уравнению баланса энергии (3.15). 


\section{4. Поведение при больших временах}

В оставшейся части работы мы будем рассматривать вязкие жидкости, описываемые системой Навье-Стокса-Фурье (2.22)-(2.25) на ограниченной регулярной области $\Omega$, где поле скоростей удовлетворяет граничному условию прилипания

$$
\left.\mathbf{u}\right|_{\partial \Omega}=0 .
$$

$\mathrm{C}$ точки зрения теории динамических систем система Навье-Стокса-Фурье обладает двумя на первый взгляд несовместимыми свойствами:

- система консервативна - полная энергия сохраняется (см. (2.6));

- система диссипативна - механическая энергия необратимо диссипирует (см. $(2.3),(2.4))$.

Для простоты мы полагаем, что единственный механизм воздействия представлен внешней объемной силой $\mathbf{f}$. Рассмотрим следующие три случая:

- "истинно" консервативный случай: $\mathbf{f}=\nabla_{x} F$, где $F=F(x)$ - независимый от времени потенциал;

- независимый от времени неконсервативный случай: $\mathbf{f}=\mathbf{f}(x) \neq \nabla_{x} F$;

- общее переменное по времени воздействие $\mathbf{f}=\mathbf{f}(t, x)$.

4.1. Равновесные решения. Как правило, равновесные решения характеризуются следующими свойствами:

- на них минимизируется производство энтропии по всем допустимым траекториям;

- на них максимизируется энтропия по всем состояниям системы;

- любая траектория стабилизируется при $t \rightarrow \infty$ вблизи своего равновесного значения.

При нашем выборе консервативных граничных условий (в частности, (4.1)) производство энтропии становится равным нулю в точке равновесия, откуда

$$
\tilde{\mathbf{u}}=0, \quad \tilde{\vartheta}=\tilde{\vartheta}(t),
$$

если $[\tilde{\varrho}, \tilde{\vartheta}, \tilde{\mathbf{u}}]-$ равновесное решение.

При данных условиях уравнение движения (1.2) сводится к уравнению

$$
\nabla_{x} p(\tilde{\varrho}, \tilde{\vartheta})=\tilde{\varrho} \mathbf{f}
$$

а из уравнения непрерывности (1.1) вытекает, что $\varrho$ = $\varrho(x)$ не зависит от времени. Поскольку полная энтропия постоянна при равновесии, то мы утверждаем следующее:

1) система допускает равновесные решения, только если $\mathbf{f}=\nabla_{x} F$, где $F=$ $F(x)$

2) любое равновесное решение имеет вид $[\tilde{\varrho}, \tilde{\vartheta}, \tilde{\mathbf{u}}=0]$, где $\tilde{\varrho}, \tilde{\vartheta}$ постоянны и не зависят от времени;

3) $\nabla_{x} p(\tilde{\varrho}, \tilde{\vartheta})=\tilde{\varrho} \nabla_{x} F$.

Соответственно, до конца этого раздела мы будем предполагать, что

$$
\mathbf{f}(t, x)=\nabla_{x} F(x), \quad F \in W^{1, \infty}\left(\Omega ; \mathbb{R}^{3}\right) .
$$


Как следствие, в системе имеются две сохраняющиеся величины:

- полная масса

$$
\int_{\Omega} \varrho(t, \cdot) \mathrm{d} x=M_{0}>0
$$

- полная энергия

$$
\int_{\Omega}\left(\frac{1}{2} \varrho|\mathbf{u}|^{2}+\varrho e(\varrho, \vartheta)-\varrho F\right)(t, \cdot) \mathrm{d} x=E_{0},
$$

где $M_{0}$ и $E_{0}$ определяются из начальных данных.

В дальнейшем мы хотим избежать равновесных плотностей $\varrho$ с вакуумом, что означает, что $\tilde{\rho}$ обращается в нуль на некотором непустом подмножестве из $\Omega$. С этой целью мы потребуем выполнения следующего дополнительного структурного ограничения на давление:

$$
\frac{\partial p(0, \tilde{\vartheta})}{\partial \varrho}>0 \quad \text { для любого } \tilde{\vartheta}>0 .
$$

В заключение данного пункта мы приведем следующий результат (см. [33; гл. 4, теорема 4.1]).

ТЕОрема 4.1. Пусть $\Omega$ - ограниченная область в $\mathbb{R}^{3}$ с липшицевой граниией. Предположим, что термодинамические функиии $p=p(\varrho, \vartheta), e=e(\varrho, \vartheta)$ непрерывно дифферениируемъ при $[\varrho, \vartheta] \in[0, \infty) \times(0, \infty)$, удовлетворяющих соотношению Гиббса (2.1), условию термодинамической устойчивости (2.2) и структурному условию (4.3). Пусть $F \in W^{1, \infty}(\Omega)$.

Тогда для заданных $M_{0}>0$ и $E_{0}$ существует не более одного липшищева равновесного решения $[\tilde{\varrho}, \tilde{\vartheta}]$ такого, что

$$
\nabla_{x} p(\tilde{\varrho}, \tilde{\vartheta})=\tilde{\varrho} \nabla_{x} F \quad{ } \Omega, \quad \int_{\Omega} \tilde{\varrho} \mathrm{d} x=M_{0}, \quad \int_{\Omega}(\tilde{\varrho} e(\tilde{\varrho}, \tilde{\vartheta})-\tilde{\varrho} F) \mathrm{d} x=E_{0} .
$$

Более того, $\tilde{\vartheta}=$ const $>0$, உ строго положительна в $\Omega$ и

$$
\int_{\Omega} \tilde{\varrho} s(\tilde{\varrho}, \tilde{\vartheta}) \mathrm{d} x \geqslant \int_{\Omega} \varrho s(\varrho, \vartheta) \mathrm{d} x
$$

для любых измеримых функиий $\varrho, \vartheta$, удовлетворяющих соотношениям

$$
\int_{\Omega} \varrho \mathrm{d} x=M_{0}, \quad \int_{\Omega}(\varrho e(\varrho, \vartheta)-\varrho F) \mathrm{d} x=E_{0} .
$$

Функционал Ляпунова и стабилизация к равновесному решению. При условии, что равновесная плотность $\varrho$ строго положительна, мы можем взять тройку $[r=\tilde{\varrho}, \Theta=\tilde{\vartheta}, \mathbf{U}=0]$ в качестве основных функций в неравенстве для относительной энтропии (3.4). Следовательно,

$$
\begin{aligned}
& {[\mathscr{E}(\varrho, \vartheta, \mathbf{u} \mid \tilde{\varrho}, \tilde{\vartheta}, 0)]_{t=0}^{t=\tau}} \\
& \quad+\int_{0}^{\tau} \int_{\Omega} \frac{\tilde{\vartheta}}{\vartheta}\left(\mathbb{S}\left(\vartheta, \nabla_{x} \mathbf{u}\right): \nabla_{x} \mathbf{u}-\frac{\mathbf{q}\left(\vartheta, \nabla_{x} \vartheta\right) \cdot \nabla_{x} \vartheta}{\vartheta}\right) \mathrm{d} x \mathrm{~d} t \leqslant 0 .
\end{aligned}
$$


Таким образом, относительная энтропия приводит к функционалу Ляпунова в потенциальном случае $\mathbf{f}=\nabla_{x} F$. В этой связи естественно возникает гипотеза, что любая траектория будет стабилизироваться к точке равновесия при $t \rightarrow \infty$. Это утверждается в следующей теореме (см. [33; гл. 4, теорема 4.5]).

Теорема 4.2. В условиях теоремы 2.1 предположим, что давление р удовлетворяет условию (4.3), и пусть $\mathbf{f}=\nabla_{x} F, F=F(x)$. Пусть $[\varrho, \vartheta, \mathbf{u}]-$ слабое решение системы Навъе-Стокса-Фуръе (2.22)-(2.25) в $[0, \infty) \times \Omega$ с полем скоростей $\mathbf{u}$, удовлетворяющим граничному условию прилипания (4.1).

Тогда

$$
\begin{aligned}
\varrho(t, \cdot) \rightarrow \tilde{\varrho} & \text { в } L^{5 / 3}(\Omega), \\
\vartheta(t, \cdot) \rightarrow \tilde{\vartheta} & \text { в } L^{4}(\Omega), \\
(\varrho \mathbf{u})(t, \cdot) \rightarrow 0 & \text { в } L^{5 / 4}\left(\Omega ; \mathbb{R}^{3}\right)
\end{aligned}
$$

при $t \rightarrow \infty$, где $[\tilde{\varrho}, \tilde{\vartheta}]$ - единственное равновесное решение, удовлетворяющее соотношениям

$$
\begin{gathered}
\int_{\Omega} \tilde{\varrho} \mathrm{d} x=\int_{\Omega} \varrho_{0} \mathrm{~d} x=M_{0} \\
\int_{\Omega}(\varrho \varrho(\tilde{\varrho}, \tilde{\vartheta})-\tilde{\varrho} F) \mathrm{d} x=\int_{\Omega}\left(\frac{1}{2} \varrho_{0}\left|\mathbf{u}_{0}\right|^{2}+\varrho_{0} e\left(\varrho_{0}, \vartheta_{0}\right)-\varrho_{0} F\right) \mathrm{d} x=E_{0} .
\end{gathered}
$$

4.2. Глобальный аттрактор в потенциальном случае. Результаты, полученные в предыдущих разделах, показывают, что множество равновесных решений представляет собой аттрактор глобальных по времени решений системы Навье-Стокса-Фурье. Точнее говоря, нашей целью является показать, что все глобальные траектории стремятся к одному из равновесных решений с равномерной скоростъю, зависящей только от размера начальных данных. Имеет место следующий результат (см. [33; гл. 5, теорема 5.1]).

ТеОрема 4.3. В условиях теоремы 4.2 рассмотрим семейство слабых решений $[\varrho, \vartheta, \mathbf{u}]$ системь Навъе-Стокса-Фурве $(2.22)-(2.25)$ в $(0, \infty) \times \Omega$, удовлетворяющих соотношению

$$
\begin{gathered}
\int_{\Omega} \varrho \mathrm{d} x \geqslant M_{0}, \quad \int_{\Omega}\left(\frac{1}{2} \varrho|\mathbf{u}|^{2}+\varrho e(\varrho, \vartheta)-\varrho F\right) \mathrm{d} x \leqslant E_{0}, \\
\underset{\tau>0}{\operatorname{essinf}} \int_{\Omega} \varrho s(\varrho, \vartheta)(\tau, \cdot) \mathrm{d} x \geqslant S_{0},
\end{gathered}
$$

где $M_{0}>0, E_{0}, S_{0}>0$ - некоторье числа.

Тогда для любого $\varepsilon>0$ существует $T=T(\varepsilon)$ такое, что

$$
\begin{gathered}
\sup _{t>T(\varepsilon)}\|\varrho(t, \cdot)-\tilde{\varrho}\|_{L^{5 / 3}(\Omega)}<\varepsilon, \\
\operatorname{ess} \sup _{t>T(\varepsilon)}\|\vartheta(t, \cdot)-\tilde{\vartheta}\|_{L^{4}(\Omega)}<\varepsilon, \\
\sup _{t>T(\varepsilon)}\|(\varrho \mathbf{u})(t, \cdot)\|_{L^{5 / 4}\left(\Omega ; \mathbb{R}^{3}\right)}<\varepsilon
\end{gathered}
$$


при условии, что слабое решение $[\varrho, \vartheta, \mathbf{u}]$ лежит в классе $(4.6)$, где $[\tilde{\varrho}, \tilde{\vartheta}]$ равновесное решение из теоремы 4.2 .

Таким образом, любое решение энергетически замкнутой диссипативной системы стабилизируется вблизи равновесного решения при больших значениях времени, что согласуется с пессимистическим следствием второго начала термодинамики:

ЭНЕРГИЯ ВСЕЛЕННОЙ ПОСТОЯННА. ЭНТРОПИЯ ВСЕЛЕННОЙ СТРЕМИТСЯ К МАКСИМУМУ.

Рудольф Клаузиус, 1822-1888

Множество равновесных решений в консервативном случае $\mathbf{f}=\nabla_{x} F(x)$ представляет собой истинный аттрактор, который притягивает множества из (4.6) с равномерной скоростью. С другой стороны, ясно, что множество всех равновесных решений неограничено.

4.3. Неконсервативные движущие силы. Теперь рассмотрим случай неконсервативных движущих сил $\mathbf{f}=\mathbf{f}(t, x)$. Мы начнем со следующих результатов, описывающих дихотомию в классе глобальных по времени решений (см. [33; гл. 5, теорема 5.2]).

Теорема 4.4. В условиях теоремы 2.1 предположим, что давление р удовлетворяет условию (4.3). Также предположим, что выполнено граничное условие прилипания (4.1). Далее, пусть задана функция

$$
\mathbf{f} \in L^{\infty}\left((0, \infty) \times \Omega ; \mathbb{R}^{3}\right) .
$$

Тогда для любого слабого решения $[\varrho, \vartheta, \mathbf{u}]$ системь Навъе-Стокса-Фуръе $(2.22)-(2.25)$ в $(0, \infty) \times \Omega$ имеет место одна из следующих альтернатив.

1. Имеет место сходимость

$$
E(\tau)=\int_{\Omega}\left(\frac{1}{2} \varrho|\mathbf{u}|^{2}+\varrho e(\varrho, \vartheta)\right)(\tau, \cdot) \mathrm{d} x \rightarrow \infty \quad \text { nрu } \tau \rightarrow \infty .
$$

2. Существует константа $E_{\infty}$ такая, что

$$
E(\tau) \leqslant E_{\infty} \quad \text { для почти всех } \tau \in(0, \infty)
$$

В последнем случае каждая последовательность времен $\tau_{n} \rightarrow \infty$ содержит подпоследовательность (которую мы отождествляем с $\left\{\tau_{n}\right\}$ ) такую, что для сдвинутых по времени функций

$$
\mathbf{f}_{n}(t, x)=\mathbf{f}\left(t+\tau_{n}, x\right)
$$

имеет место сходимость

$$
\mathbf{f}_{n} \rightarrow \nabla_{x} F \quad \text { *-слабо в } L^{\infty}\left((0, T) \times \Omega ; \mathbb{R}^{3}\right), \quad T>0-\text { произвольное }
$$


где предельный потенииал

$$
F=F(x), \quad F \in W^{1, \infty}(\Omega),
$$

может зависеть от выбора последовательности $\left\{\tau_{n}\right\}_{n=1}^{\infty}$.

Непосредственным следствием теоремы 4.4 является следующий результат.

СлЕДСТвиЕ 4.1. В условиях теоремы 4.4 предположим, что

$$
\mathbf{f}=\mathbf{f}(x), \quad \mathbf{f} \neq \nabla_{x} F .
$$

Тогда

$$
E(\tau)=\int_{\Omega}\left(\frac{1}{2} \varrho|\mathbf{u}|^{2}+\varrho e(\varrho, \vartheta)\right)(\tau, \cdot) \mathrm{d} x \rightarrow \infty \quad \text { npu } \tau \rightarrow \infty
$$

для любого решения $[\varrho, \vartheta, \mathbf{u}]$ системы Навъе-Стокса-Фуръе, на которую дейcmвуеm $\mathbf{f}$.

Интуитивно понятно, что альтернатива (4.7) с "расходящейся" энергией имеет место, если механическая энергия, вносимая в систему через f, необратимо превращается в тепло. Как следствие, можно ожидать, что компонента внутренней энергии тоже становиться бесконечной. Это будет действительно так, если мы слегка усилим наши предположения о коэффициенте сдвиговой (динамической) вязкости $\mu$ (см. [33; гл. 5, следствие 5.3]).

Теорема 4.5. В условиях теоремы 4.3 предположим, что коэфбиииент сдвиговой вязкости н является непрерьвно дифференцируемой функцией от $\vartheta$ такой, что

$$
0<\underline{\mu}(1+\vartheta) \leqslant \mu(\vartheta), \quad\left|\mu^{\prime}(\vartheta)\right|<c \quad \text { для всех } \vartheta \in[0, \infty) .
$$

Тогда для любого слабого решения $[\varrho, \vartheta, \mathbf{u}]$ системы Навъе-Стокса-Фуръе $(2.22)-(2.25)$ в $(0, \infty) \times \Omega$ имеют место следующие альтернативы.

1. $\int_{\Omega} \varrho e(\varrho, \vartheta)(\tau, \cdot) \mathrm{d} x \rightarrow \infty$ npu $\tau \rightarrow \infty$.

2. Существует константа $E_{\infty}$ такая, что

$$
\int_{\Omega}\left(\frac{1}{2} \varrho|\mathbf{u}|^{2}+\varrho e(\varrho, \vartheta)\right)(\tau, \cdot) \mathrm{d} x \leqslant E_{\infty} \quad \text { для почти всех } \tau \in(0, \infty) .
$$

4.3.1. Решения с неограниченной энергией. Используя теорему 4.4, мы можем легко построить примеры движущих сил, для которых энергия расходится, т. е.

$$
\int_{\Omega}\left(\frac{1}{2} \varrho|\mathbf{u}|^{2}+\varrho e(\varrho, \vartheta)\right)(\tau, \cdot) \mathrm{d} x \rightarrow \infty \quad \text { при } \tau \rightarrow \infty
$$

(по поводу деталей мы отсылаем читателя к [33; гл. $5, \S 5.2 .2])$ :

1) f почти периодична (периодична) по $t$, но не постоянна по $t$;

2) f асимптотически почти периодична (периодична), т. е.

$$
\sup _{x \in \Omega}|\mathbf{f}(t, x)-\mathbf{g}(t, x)| \rightarrow 0 \quad \text { при } t \rightarrow \infty,
$$

где $\mathbf{g}$ почти периодична, но не постоянна по времени; 
3) для любого непустого открытого подмножества $B \subset \Omega$ существует $\mathbf{f} \in$ $C^{\infty}\left((0, T) \times \Omega ; \mathbb{R}^{3}\right)$ такая, что

$$
\begin{gathered}
\operatorname{supp}[\mathbf{f}(t, \cdot)] \subset B \quad \text { для любого } t>0, \\
\|\mathbf{f}(t, \cdot)\|_{C^{m}\left(\Omega ; \mathbb{R}^{3}\right)} \rightarrow 0 \quad \text { при } t \rightarrow \infty, \quad m=1,2, \ldots,
\end{gathered}
$$

и

$$
\int_{\Omega}\left(\frac{1}{2} \varrho|\mathbf{u}|^{2}+\varrho e(\varrho, \vartheta)\right)(\tau, \cdot) \mathrm{d} x \rightarrow \infty \quad \text { при } \tau \rightarrow \infty
$$

для любого решения системы Навье-Стокса-Фурье, на которую действует $\mathbf{f}$.

4.4. Системы, на которые действуют осциллирующие силы. В свете сказанного выше может показаться, что почти "любая" внешняя сила $\mathbf{f}$ делает энергию энергетически изолированной жидкой системы бесконечной, за исключением случая, когда $\mathbf{f}$ является градиентом независимого от времени скалярного потенциала. Тем не менее при выполнении второй альтернативы из теоремы 4.4 возможны некоторые интересные исключения, например, быстро осциллирующие движущие силы, которые мы обсудим в данном пункте.

Для стандартной двумерной несжимаемой системы Навье-Стокса, на которую действует внешняя сила вида

$$
\mathbf{f}=\mathbf{g}_{1}+\varepsilon^{-\alpha} \mathbf{g}_{2}\left(\frac{t}{\varepsilon}\right)
$$

Чепыжов, Пата и Вишик [10] исследовали ее поведение при больших временах и аттракторы. Ими было показано, что так называемые траекторные аттракторы $\mathscr{A}_{\varepsilon}$ сходятся в определенном смысле при $\varepsilon \rightarrow 0$ к аттрактору $\mathscr{A}_{0}$ системы, на которую действует $\mathbf{g}_{1}$. Таким образом, сильно осциллирующие возмущения не оказывают влияния на асимптотическое поведение решений, даже несмотря на то, что их амплитуда может быть весьма большой.

Следуя данному подходу, мы рассмотрим неограниченные (в общем случае) движущие силы вида

$$
\mathbf{f}(t, x)=t^{\delta} \omega\left(t^{\beta}\right) \mathbf{w}(x),
$$

где $\omega$ - ограниченная функция с равномерно ограниченными средними по времени (см. условие (4.10) в приводимой ниже теореме).

Имеет место следующий результат [7; теорема 1].

Теорема 4.6. В условиях теоремы 2.1 предположим, что

$$
\mathbf{f}(t, x)=t^{\delta} \omega\left(t^{\beta}\right) \mathbf{w}(x),
$$

əəe

$$
\omega \in L^{\infty}(\mathbb{R}), \quad \omega \neq 0, \quad \sup _{\tau>0}\left|\int_{0}^{\tau} \omega(t) \mathrm{d} t\right|<\infty,
$$

а параметры $\beta, \delta$ таковы, что

$$
\delta>0, \beta>2+2 \delta \quad \text { или } \delta \leqslant 0, \beta>2+\delta .
$$


Тогда для любого слабого решения $[\varrho, \vartheta, \mathbf{u}]$ системы Навъе-Стокса-Фуръе $(2.22)-(2.25)$ в $(0, \infty) \times \Omega$ имеет место сходимость

$$
\begin{array}{rlrl}
\varrho(t, \cdot) & \rightarrow \tilde{\varrho} & \text { в } L^{5 / 3}(\Omega) & \text { при } t \rightarrow \infty, \\
\vartheta(t, \cdot) \rightarrow \tilde{\vartheta} & \text { в } L^{4}(\Omega) & \text { nри } t \rightarrow \infty, \\
(\varrho \mathbf{u})(t, \cdot) \rightarrow 0 & \text { в } L^{5 / 4}\left(\Omega ; \mathbb{R}^{3}\right) & & \text { nри } t \rightarrow \infty,
\end{array}
$$

где $\tilde{\varrho}, \tilde{\vartheta}$ - положительные постоянные,

$$
\tilde{\varrho}=\frac{1}{|\Omega|} \int_{\Omega} \varrho \mathrm{d} x .
$$

ПримечАниЕ 4.1. По-видимому, условие (4.11) не является оптимальным. С другой стороны, некоторое ускорение осцилляций все же необходимо, поскольку в противном случае мы бы пришли к ситуации, описанной в (4.7).

\section{5. Примеры поведения при больших временах для неконсервативных систем. Выводы}

Для неконсервативных жидких систем, в которые энергия подводится или в которых происходит ее обмен с внешним "миром", имеется больше интересных возможностей асимптотического поведения, чем в рассмотренном нами случае энергетически изолированных систем.

5.1. Периодическое по времени движение. Следуя [29], рассмотрим систему Навье-Стокса-Фурье, на которую действует периодическая по времени внешняя сила $\mathbf{f}$,

$$
\mathbf{f} \in L^{\infty}\left(\mathbb{R} \times \Omega ; \mathbb{R}^{3}\right), \quad \mathbf{f}(t+\omega ; \cdot)=\mathbf{f}(t, \cdot), \omega>0, \text { для почти всех } t \in \mathbb{R} .
$$

Аналогично предыдущему разделу, рассмотрим граничное условие прилипания для скорости

$$
\left.\mathbf{u}\right|_{\partial \Omega}=0 .
$$

Для получения нетривиальных результатов мы заменим консервативное условие отсутствия потока (1.6) на следующее:

$$
\left.\mathbf{q} \cdot \mathbf{n}\right|_{\partial \Omega}=\left.d\left(\vartheta-\Theta_{0}\right)\right|_{\partial \Omega}, \quad d \in L^{\infty}(\partial \Omega), \quad \Theta_{0} \in L^{1}(\partial \Omega) .
$$

В отличие от (1.6) граничные условия (5.3) допускают обмен тепловой энергии через границу, что может препятствовать выполнению альтернативы (4.7).

5.1.1. Слабая формулировка. Для того чтобы учесть периодичность решений по времени, а также граничные условия (5.3), мы должны модифицировать слабую формулировку (2.22)-(2.25).

Пусть

$$
S^{1}=\left.[0, \omega]\right|_{\{0, \omega\}}
$$

- "плоская" сфера по переменной времени. Мы говорим, что $[\varrho, \vartheta, \mathbf{u}]-$ nериодическое по времени слабое решение системы Навье-Стокса-Фурье с периодом $\omega>0$, если выполнены следующие условия: 
- плотность и температура положительны, $\varrho(t, x) \geqslant 0, \vartheta(t, x)>0$ для почти всех $(t, x) \in S^{1} \times \Omega$,

$$
\begin{array}{lll}
\varrho \in C_{\text {weak }}\left(S^{1} ; L^{5 / 3}\right), & (\varrho \mathbf{u}) & \in C_{\text {weak }}\left(S^{1} ; L^{\beta}\left(\Omega ; \mathbb{R}^{3}\right)\right), \\
\vartheta & \in L^{\infty}\left(S^{1} ; L^{4}(\Omega)\right) \cap L^{2}\left(S^{1} ; W^{1,2}(\Omega)\right), & \mathbf{u} \in L^{2}\left(S^{1} ; W_{0}^{1,2}\left(\Omega ; \mathbb{R}^{3}\right)\right) ;
\end{array}
$$

- уравнение непрерывности имеет вид

$$
\int_{S^{1}} \int_{\Omega}\left[(\varrho+b(\varrho)) \partial_{t} \varphi+(\varrho+b(\varrho)) \mathbf{u} \cdot \nabla_{x} \varphi+\left(b(\varrho)-b^{\prime}(\varrho) \varrho\right) \operatorname{div}_{x} \mathbf{u} \varphi\right] \mathrm{d} x \mathrm{~d} t=0
$$

для любой основной функции $\varphi \in C^{1}\left(S^{1} \times \bar{\Omega}\right)$ и любого $b \in C^{1}[0, \infty)$ такого, что $b(0)=0, b^{\prime}(\varrho)=0$ при всех $\varrho \geqslant c_{b} ;$

- уравнение движения выполняется в смысле обобщенных функций:

$$
\int_{S^{1}} \int_{\Omega}\left(\varrho \mathbf{u} \cdot \partial_{t} \varphi+\varrho \mathbf{u} \otimes \mathbf{u}: \nabla_{x} \varphi+p(\varrho, \vartheta) \operatorname{div}_{x} \varphi-\mathbb{S}: \nabla_{x} \varphi+\varrho \mathbf{f} \cdot \varphi\right) \mathrm{d} x \mathrm{~d} t=0
$$

для любой $\varphi \in C^{1}\left(S^{1} \times \bar{\Omega} ; \mathbb{R}^{3}\right)$, где $\left.\varphi\right|_{\partial \Omega}=0$;

- уравнение баланса энтропии заменяется семейством интегральных неравенств

$$
\begin{array}{r}
\int_{S^{1}} \int_{\Omega} \frac{\varphi}{\vartheta}\left(\mathbb{S}: \nabla_{x} \mathbf{u}-\frac{\mathbf{q} \cdot \nabla_{x} \vartheta}{\vartheta}\right) \mathrm{d} x \mathrm{~d} t-\int_{S^{1}} \int_{\partial \Omega} \frac{d}{\vartheta}\left(\vartheta-\Theta_{0}\right) \varphi \mathrm{d} S_{x} \\
\leqslant-\int_{S^{1}} \int_{\Omega}\left(\varrho s(\varrho, \vartheta) \partial_{t} \varphi+\varrho s(\varrho, \vartheta) \mathbf{u} \cdot \nabla_{x} \varphi+\frac{\mathbf{q} \cdot \nabla_{x} \varphi}{\vartheta}\right) \mathrm{d} x \mathrm{~d} t
\end{array}
$$

для любой $\varphi \in C^{1}\left(S^{1} \times \bar{\Omega}\right), \varphi \geqslant 0$;

- уравнение полной энергии записывается как

$$
\begin{aligned}
& \int_{S^{1}} {\left[\partial_{t} \psi \int_{\Omega}\left(\frac{1}{2} \varrho|\mathbf{u}|^{2}+\varrho e(\varrho, \vartheta)\right) \mathrm{d} x\right] \mathrm{d} t } \\
& \quad=\int_{S^{1}} \psi\left[\int_{\partial \Omega} d\left(\vartheta-\Theta_{0}\right) \mathrm{d} S_{x}-\int_{\Omega} \varrho \mathbf{f} \cdot \mathbf{u} \mathrm{d} x\right] \mathrm{d} t
\end{aligned}
$$

для любой $\psi \in C^{\infty}\left(S^{1}\right)$.

5.1.2. Существование периодических по времени решений. Следующий результат доказан в [29; теорема 2.1].

Теорема 5.1. В условиях теоремы 4.3 предположим, что коэфбиииент сдвиговой вязкости н является непрерывно дифференцируемой функиией от ७, удовлетворяющей условиям

$$
0<\underline{\mu}(1+\vartheta) \leqslant \mu(\vartheta), \quad\left|\mu^{\prime}(\vartheta)\right|<c \quad \text { для всех } \vartheta \in[0, \infty) .
$$


Пусть f удовлетворяет условиям (5.1), и пусть функиии d, $\Theta_{0}$ из (5.3) удовлетворяют неравенствам

$$
d(x) \geqslant \underline{d}>0, \quad \Theta_{0}(x) \geqslant \underline{\Theta}>0 \quad \text { для почти всех } x \in \partial \Omega .
$$

Тогда для каждого $M_{0}>0$ система Навъе-Стокса-Фуръе (5.4)-(5.7) допускает периодическое по времени слабое решение $[\varrho, \vartheta, \mathbf{u}]$ такое, что

$$
\int_{\Omega} \varrho \mathrm{d} x=M_{0} .
$$

Для задач с неконсервативными граничными условиями можно ожидать аналогичных результатов, в частности существования ограниченных притягивающих множеств и глобальных аттракторов.

5.2. Заключительные комментарии. В соответствии со сказанным выше, при больших временах динамика энергетически изолированных жидких систем достаточно проста и понятна. Неизбежным следствием второго закона термодинамики является то, что любая система под действием консервативной внешней силы стремится при $t \rightarrow \infty$ к единственному равновесному решению, однозначно определяемому общей массой и энергией жидкости. В случае, если на жидкость действует неконсервативная сила, но ее физическая граница все еще остается изолированной, то энергия необратимо переходит в тепло, следствием чего является расходимость полной энергии системы при бесконечном времени. Достаточно интересно, что при быстро осциллирующих внешних силах такой сценарий не обязателен - под действием такой силы система может иметь единственное равновесное решение с конечной энергией.

Полученные результаты имеют достаточно общий характер и применимы к широкому классу слабых решений без каких-либо ограничений на размер начальных данных. С другой стороны, не следует забывать, что технические предположения о специальном виде определяющих уравнений были продиктованы нуждами обеспечения существования для теории, поэтому можно надеяться, что многие из предыдущих результатов будут верны и в более общей ситуации.

Далее, выше показано, что в соответствии со вторым законом термодинамики энергетически изолированные жидкие системы эволюционируют к точно определенным и полностью предсказуемым состояниям. Этот вывод, однако, является достаточно неожиданным и резко отличается от общепринятого сценария, согласно которому аттракторы со сложной топологической структурой являются объектами, "правильно схватывающими” характерные черты поведения жидких систем (см., например, работу Экманна и Рюэлля [21]). Для осмысления такого явного "парадокса" следует привлечь следующие аргументы.

- В реальном мире временнь́е шкалы, связанные с турбулентностью, значительно короче, чем то "бесконечное" время, за которое в соответствии со вторым началом термодинамики происходит тепловая смерть вселенной. Не менее важно также и то, что настоящая теория не ограничена принципами классической механики и, как следствие, любые метафизические заключения такого типа определенно находятся за пределами достижимого данным методом. 
- Жидкости могут переносить тепло. Однако та часть кинетической энергии жидкости, которая необратимо диссипирует, достаточно мала или даже пренебрежимо мала в сравнении с эффектами конвекции. К этому следует добавить, что теплопроводность некоторых жидкостей (газов) может быть очень малой.

- Не существует идеально изолированных от внешнего мира физических систем. Наоборот, для многих диссипативных систем характеристическим свойством является то, что они эффективно избавляются от энтропии через внешнее "охлаждение", как в примере периодического по времени движения, которое обсуждалось в п. 5.1 .

- Тепловые и механические воздействия могут быть независимыми, по крайней мере на наблюдаемых временных шкалах, что приводит к изотермическим или изэнтропическим системам, в которых механическое движение полностью изолировано от тепловых эффектов.

\section{Список литературы}

[1] T. Alazard, "Low Mach number flows and combustion", SIAM J. Math. Anal., 38:4 (2006), 1186-1213 (electronic).

[2] С.Н. Антонцев, А.В. Кажихов, В.Н. Монахов, Краевые задачи механики неоднородных жидкостей, Наука, Новосибирск, 1983, 320 с.; англ. пер.: S. N. Antontsev, A.V. Kazhikhov, V.N. Monakhov, Boundary value problems in mechanics of nonhomogeneous fluids, Stud. Math. Appl., 22, North-Holland, Amsterdam, 1990, xii+309 pp.

[3] А.В. Бабин, М.И. Вишик, Аттракторы эволючионнъх уравнений, Наука, М., 1989, 296 с.; англ. пер.: A. V. Babin, M. I. Vishik, Attractors of evolution equations, Stud. Math. Appl., 25, North-Holland, Amsterdam, 1992, x+532 pp.

[4] E. Becker, Gasdynamik, Leitfäden Angew. Math. Mech., 6, B. G. Teubner Verlagsgesellschaft, Stuttgart, 1966, 248 pp.

[5] F. Belgiorno, "Notes on the third law of thermodynamics. I", J. Phys. A, 36:30 (2003), 8165-8193.

[6] F. Belgiorno, "Notes on the third law of thermodynamics. II", J. Phys. A, 36:30 (2003), 8195-8221.

[7] P. Bella, E. Feireisl, D. Pražák, "Long time behavior and stabilization to equilibria of solutions to the Navier-Stokes-Fourier system driven by highly oscillating unbounded external forces", J. Dynam. Differential Equations, 25:2 (2013), 257-268.

[8] L. Caffarelli, R. V. Kohn, L. Nirenberg, "Partial regularity of suitable weak solutions of the Navier-Stokes equations", Commun. Pure Appl. Math., 35:6 (1982), 771-831.

[9] H. B. Callen, Thermodynamics and an introduction to thermostatistics, 2nd ed., Wiley, New York, 1985, 512 pp.

[10] V. V. Chepyzhov, V. Pata, M. I. Vishik, "Averaging of 2D Navier-Stokes equations with singularly oscillating forces", Nonlinearity, 22:2 (2009), 351-370.

[11] V. V. Chepyzhov, M. I. Vishik, "Evolution equations and their trajectory attractors", J. Math. Pures Appl. (9), 76:10 (1997), 913-964.

[12] E. Chiodaroli, E. Feireisl, O. Kreml, On the weak solutions to the equations of a compressible heat conducting gas, 2013, 27 pp., arXiv: 1307.0640; Ann. Inst. H. Poincaré Anal. Non Linéaire (to appear).

[13] P. Constantin, C. Foias, Navier-Stokes equations, Chicago Lectures in Math., Univ. of Chicago Press, Chicago, IL, 1988, x+190 pp. 
[14] P. Constantin, C. Foias, B. Nicolaenko, R. Temam, Integral manifolds and inertial manifolds for dissipative partial differential equations, Appl. Math. Sci., 70, Springer-Verlag, New York, 1989, $\mathrm{x}+123$ pp.

[15] P. Constantin, C. Foias, R. Temam, Attractors representing turbulent flows, Mem. Amer. Math. Soc., 53, № 314, 1985, vii+67 pp.

[16] C. M. Dafermos, "The second law of thermodynamics and stability", Arch. Ration. Mech. Anal., 70:2 (1979), 167-179.

[17] C. De Lellis, L. Székelyhidi, Jr., "The Euler equations as a differential inclusion", Ann. of Math. (2), 170:3 (2009), 1417-1436.

[18] C. De Lellis, L. Székelyhidi, Jr., "On admissibility criteria for weak solutions of the Euler equations", Arch. Ration. Mech. Anal., 195:1 (2010), 225-260.

[19] C. De Lellis, L. Székelyhidi, Jr., "The $h$-principle and the equations of fluid dynamics", Bull. Amer. Math. Soc. (N. S.), 49:3 (2012), 347-375.

[20] R. J. DiPerna, P.-L. Lions, "Ordinary differential equations, transport theory and Sobolev spaces", Invent. Math., 98:3 (1989), 511-547.

[21] J.-P. Eckmann, D. Ruelle, "Ergodic theory of chaos and strange attractors", Rev. Modern Phys., 57:3, part 1 (1985), 617-656.

[22] S. Eliezer, A. Ghatak, H. Hora, An introduction to equations of state: theory and applications, Cambridge Univ. Press, Cambridge, 1986, 384 pp.

[23] J. L. Ericksen, Introduction to the thermodynamics of solids, rev. ed., Appl. Math. Sci., 131, Springer-Verlag, New York, 1998, xii+189 pp.

[24] J. Fan, S. Jiang, Y. Ou, "A blow-up criterion for compressible viscous heat-conductive flows", Ann. Inst. H. Poincaré Anal. Non Linéaire, 27:1 (2010), 337-350.

[25] C.L. Fefferman, "Existence and smoothness of the Navier-Stokes equation", The millennium prize problems, Clay Math. Inst., Cambridge, MA, 2006, 57-67.

[26] E. Feireisl, "On compactness of solutions to the compressible isentropic Navier-Stokes equations when the density is not square integrable", Comment. Math. Univ. Carolin., 42:1 (2001), 83-98.

[27] E. Feireisl, "On the motion of a viscous, compressible, and heat conducting fluid", Indiana Univ. Math. J., 53:6 (2004), 1707-1740.

[28] E. Feireisl, "Relative entropies in thermodynamics of complete fluid systems", Discrete Contin. Dyn. Syst., 32:9 (2012), 3059-3080.

[29] E. Feireisl, P. B. Mucha, A. Novotný, M. Pokorný, "Time-periodic solutions to the full Navier-Stokes-Fourier system", Arch. Ration. Mech. Anal., 204:3 (2012), 745-786.

[30] E. Feireisl, A. Novotný, Singular limits in thermodynamics of viscous fluids, Adv. Math. Fluid Mech., Birkhäuser Verlag, Basel, 2009, xxxvi+382 pp.

[31] E. Feireisl, A. Novotný, "Weak-strong uniqueness property for the full Navier-StokesFourier system", Arch. Ration. Mech. Anal., 204:2 (2012), 683-706.

[32] E. Feireisl, A. Novotný, Y. Sun, "A regularity criterion for the weak solutions to the Navier-Stokes-Fourier system", Arch. Ration. Mech. Anal., 212:1 (2014), 219-239.

[33] E. Feireisl, D. Pražák, Asymptotic behavior of dynamical systems in fluid mechanics, AIMS Ser. Appl. Math., 4, American Institute of Mathematical Sciences (AIMS), Springfield, MO, 2010, xii+298 pp.

[34] C. Foias, O. Manley, R. Rosa, R. Temam, Navier-Stokes equations and turbulence, Encyclopedia Math. Appl., 83, Cambridge Univ. Press, Cambridge, 2001, xiv+347 pp.

[35] G. Gallavotti, Statistical mechanics. A short treatise, Texts Monogr. Phys., SpringerVerlag, Berlin, 1999, xiv+339 pp.

[36] G. Gallavotti, Foundations of fluid dynamics, Texts Monogr. Phys., Springer-Verlag, Berlin, 2002, xviii+513 pp.

[37] P. Germain, "Weak-strong uniqueness for the isentropic compressible Navier-Stokes system", J. Math. Fluid Mech., 13:1 (2011), 137-146. 
[38] S. Jiang, Y. Ou, "A blow-up criterion for compressible viscous heat-conductive flows", Acta Math. Sci. Ser. B Engl. Ed., 30:6 (2010), 1851-1864.

[39] T. Kato, "Remarks on the zero viscosity limit for nonstationary Navier-Stokes flows with boundary", Seminar on nonlinear partial differential equations, Berkeley, CA, 1983, Math. Sci. Res. Inst. Publ., 2, ed. S. S. Chern, Springer, New York, 1984, 85-98.

[40] O. A. Ladyzhenskaya, The mathematical theory of viscous incompressible flow, 2nd ed., Mathematics and its Applications, 2, Gordon and Breach, New York-London-Paris, 1969, xviii+224 с.; 1-е рус. изд.: О. А. Ладыженская, Математические вопросы динамики вязкой несжимаемой жидкости, Физматгиз, М., 1961.

[41] О.А. Ладыженская, "Об однозначной разрешимости в целом трехмерной задачи Коши для уравнений Навье-Стокса при наличии осевой симметрии", Kраевые задачи математической физики и смежные вопросы теории функиий. 2, Зап. науч. сем. ЛОМИ, 7, Изд-во "Наука", Ленинград. отд., Л., 1968, 155-177.

[42] О.А. Ладыженская, "О конечномерности ограниченных инвариантных множеств для системы Навье-Стокса и других диссипативных систем", Краевые задачи математической физики и смежные вопросы теории функиий. 14, Зап. науч. сем. ЛОМИ, 115, Изд-во "Наука", Ленинград. отд., Л., 1982, 137-155; англ. пер.: О. A. Ladyzhenskaya, "Finite-dimensionality of bounded invariant sets for Navier-Stokes systems and other dissipative systems", J. Soviet Math., 28:5 (1985), $714-726$.

[43] J. Leray, "Sur le mouvement d'un liquide visqueux emplissant l'espace", Acta Math., 63:1 (1934), 193-248.

[44] P.-L. Lions, Mathematical topics in fluid mechanics, v. 1: Incompressible models, Oxford Lecture Ser. Math. Appl., 3, The Clarendon Press, Oxford Univ. Press, New York, 1996, xiv+237 pp.

[45] P.-L. Lions, Mathematical topics in fluid mechanics, v. 2: Compressible models, Oxford Lecture Ser. Math. Appl., 10, The Clarendon Press, Oxford Univ. Press, New York, 1998, xiv+348 pp.

[46] A. Matsumura, T. Nishida, "The initial value problem for the equations of motion of viscous and heat-conductive gases", J. Math. Kyoto Univ., 20:1 (1980), 67-104.

[47] A. Matsumura, T. Nishida, "Initial boundary value problems for the equations of motion of compressible viscous and heat-conductive fluids", Comm. Math. Phys., 89:4 (1983), 445-464.

[48] Y. Qin, Nonlinear parabolic-hyperbolic coupled systems and their attractors, Oper. Theory Adv. Appl., 184, Adv. Partial Differ. Equ. (Basel), Birkhäuser Verlag, Basel, 2008, xvi+465 pp.

[49] D. Serre, "The structure of dissipative viscous system of conservation laws", Phys. D, 239:15 (2010), 1381-1386.

[50] D. Serre, "Viscous system of conservation laws: singular limits", Nonlinear conservation laws and applications, IMA Vol. Math. Appl., 153, Springer, New York, 2011, 433-445.

[51] T. Tao, Nonlinear dispersive equations. Local and global analysis, CBMS Reg. Conf. Ser. Math., 106, Amer. Math. Soc., Providence, RI, 2006, xvi+373 pp.

[52] R. Temam, Navier-Stokes equations. Theory and numerical analysis, Stud. Math. Appl., 2, North-Holland, Amsterdam-New York-Oxford, 1977, x+500 pp.

[53] R. Temam, Infinite-dimensional dynamical systems in mechanics and physics, Appl. Math. Sci., 68, Springer-Verlag, New York, 1988, xvi+500 pp.

[54] A. Valli, "An existence theorem for compressible viscous fluids", Ann. Mat. Pura Appl. (4), 130:1 (1982), 197-213.

[55] A. Valli, "A correction to the paper: An existence theorem for compressible viscous fluids", Ann. Mat. Pura Appl. (4), 132:1 (1982), 399-400. 
[56] A. Valli, M. Zajaczkowski, "Navier-Stokes equations for compressible fluids: Global existence and qualitative properties of the solutions in the general case", Comm. Math. Phys., 103:2 (1986), 259-296.

[57] C. H. Wilcox, Sound propagation in stratified fluids, Appl. Math. Sci., 50, SpringerVerlag, New York, 1984, ix+198 pp.

\section{Эдуард Файрайзл}

(Eduard Feireisl)

Mathematical Institute, Academy of Sciences

of the Czech Republic

E-mail: feireisl@math.cas.cz
Поступила в редакцию 27.10 .2013 